INPLASY

PROTOCOL

To cite: Zhang et al. The Outcome of The On- Versus Off- Pump Coronary Artery By Pass Grafting: A MetaAnalysis. Inplasy protocol 202080089. doi:

10.37766/inplasy2020.8.0089

Received: 22 August 2020

Published: 22 August 2020

Corresponding author: Chen Liangwan

chenliangwan@tom.com

Author Affiliation:

Department of Cardiac

Surgery, Fujian Medical

University Union Hospital,

Fuzhou, China

Support: None

Review Stage at time of this submission: Preliminary searches.

Conflicts of interest:

There is no conflict of interest because there is no sponsor for this article.

\section{The Outcome of The On- Versus Off- Pump Coronary Artery By Pass Grafting: A Meta-Analysis}

Zhang, Y1; Chai, T2; He, J3; Chen, L4.

Review question / Objective: The aim of this meta-analysis is to investigate the different in outcome of the on-pump with off-pump CABG.

Condition being studied: Coronary artery bypass grafting (CABG) is the gold standard treatment for the patients with extensive coronary artery disease. Due to a series of complications associated with on-pump CABG, Off-pump bypass surgery was introduced, but the outcomes were mixed.

Information sources: Our information sources will from four electronic debases including PubMed (MEDLINE), Cochrane Library, Web of Science, ClinicalTrials.gov. If the data is not clear in the publication, we will contact the author.

INPLASY registration number: This protocol was registered with the International Platform of Registered Systematic Review and Meta-Analysis Protocols (INPLASY) on 22 August 2020 and was last updated on 22 August 2020 (registration number INPLASY202080089).

\section{INTRODUCTION}

Review question / Objective: The aim of this meta-analysis is to investigate the different in outcome of the on-pump with off-pump CABG.
Condition being studied: Coronary artery bypass grafting (CABG) is the gold standard treatment for the patients with extensive coronary artery disease. Due to a series of complications associated with on- 
pump CABG, Off-pump bypass surgery was introduced, but the outcomes were mixed.

\section{METHODS}

Participant or population: The patients both male or female adult ( $\geq 18$ years) with coronary artery disease who were undergoing CABG either off or on-pump.

Intervention: Patients with stable angina or acute coronary syndrome being treated with CABG were exposed to either offpump or on-pump.

Comparator: The studies in this analysis will be compared the outcomes of offpump CABG with a usual-care control group receiving on -pump CABG.

Study designs to be included: Only randomized controlled trials (RCTs) and their studies where this did not involve duplication of data of off-pump vs. onpump in patients undergoing CABG will be included. All English publications until June 2020 will be searched without the restrictions of national or regional.

Eligibility criteria: All identified papers will be assessed independently by two reviewers. A third reviewer will be consulter to resolve disputes.

Information sources: Our information sources will from four electronic debases including PubMed (MEDLINE), Cochrane Library, Web of Science, ClinicalTrials.gov. If the data is not clear in the publication, we will contact the author.

Main outcome(s): We will set stroke as the main outcome.

Quality assessment / Risk of bias analysis: Two reviewers will independently assess the quality of the selected studies according to the Cochrane Collaboration's tool for randomized controlled trials. Items will be evaluated in three categories: Low risk of bias, unclear bias and high risk of bias. The following characteristics will be evaluated: Random sequence generation (selection bias) Allocation concealment (selection bias) Blinding of participants and personnel (performance bias) Incomplete outcome data (attrition bias) Selective reporting (reporting bias) Other biases. Results from these questions will be graphed and assessed using Review Manager 5.3.

Strategy of data synthesis: Odds ratios [ORs] will be calculated for dichotomous data. Mean differences will be calculated for continuous data. All analyses will be conducted using Review Manager 5.3 (Nordic Cochrane Centre, Copenhagen, Denmark). A fixed effect inverse variance model will be used unless heterogeneity was $>50 \%$, then a random effects model was used. Heterogeneity was quantified using the Cochrane $Q$ test. We used a $5 \%$ level of significance and $95 \%$ confidence intervals; figures were produced using Review Manager 5.3.

Subgroup analysis: If $12 \geq 75 \%$, we will consider subgroups such as age, clinical type.

Sensibility analysis: The main methods of sensitivity analysis include changing the inclusion criteria (especially controversial studies), excluding low-quality studies, and using different statistical methods/models to analyze the same data.

Country(ies) involved: China.

Keywords: Coronary artery bypass grafting, off-pump and on-pump.

Contributions of each author:

Author 1 - Zhang Yuling.

Author 2 - Chai Tianci.

Author 3 - He Jian.

Author 4 - Chen Liangwan. 